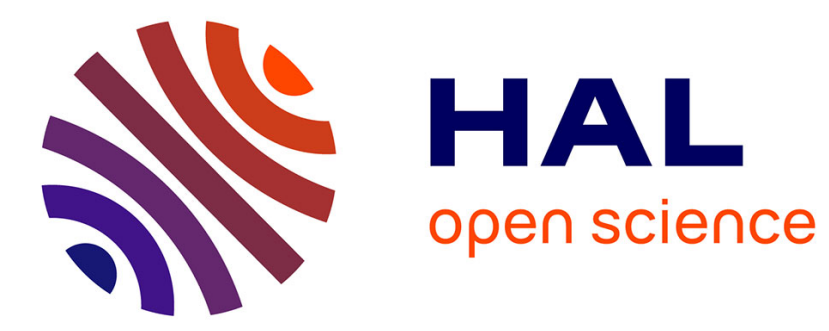

\title{
Des formules au nom : bases pour une théorie de la sexuation chez Lacan
}

\author{
Pedro Eduardo Silva Ambra
}

\section{To cite this version:}

Pedro Eduardo Silva Ambra. Des formules au nom : bases pour une théorie de la sexuation chez Lacan. In Analysis, 2018, 2 (1), pp.69-70. 10.1016/j.inan.2018.02.006 . hal-02423609

\section{HAL Id: hal-02423609 \\ https://hal.science/hal-02423609}

Submitted on 8 Jan 2020

HAL is a multi-disciplinary open access archive for the deposit and dissemination of scientific research documents, whether they are published or not. The documents may come from teaching and research institutions in France or abroad, or from public or private research centers.
L'archive ouverte pluridisciplinaire HAL, est destinée au dépôt et à la diffusion de documents scientifiques de niveau recherche, publiés ou non, émanant des établissements d'enseignement et de recherche français ou étrangers, des laboratoires publics ou privés. 
AMBRA, P.. « Des formules au nom : bases pour une théorie de la sexuation chez Lacan ». In Analysis, v. 2, p. 69-70, 2018. Doi: 10.1016/j.inan.2018.02.006

RESUME DE LA THESE - Des formules au nom : bases pour une théorie de la sexuation chez Lacan

Pedro Ambra

Cette thèse a eu pour but la construction des bases conceptuelles qui peuvent soutenir une nouvelle lecture de la théorie de la sexuation chez Lacan. Le texte cherche à extraire les conséquences de l'aphorisme "l'être sexué ne s'autorise que de lui-même et de quelques autres ", énoncé au sein d'une description tardive des formules de la sexuation. Nous partons du constat d'un hiatus entre les expressions contemporaines des sexualités et la portée des outils théoriques lacaniens les plus hégémoniques dans ce champ. Si nous défendons que les réflexions au sein du lacanisme n'ont pas tout dit sur ce sujet, la thèse essaie de montrer en quoi les textes de Lacan permettent de penser plus loin que ce que leurs diverses interprétations ont soutenu jusque-là.

Cette perspective de recherche est abordée en prenant la notion d'identification comme fil conducteur, puisqu'elle permet de dialectiser deux pôles de la théorie psychanalytique : d'un côté, le noyau pulsionnel, pas-tout et contingent (c'est-à-dire le caractère ineffable de l'inconscient en tant qu'instance majeure d'une vérité subjective inconnu, mais déterminante) ; de l'autre, celui d'une nécessaire aliénation du sujet (le moi comme l'illusion d'une unité, énonçable grâce à ses identités). L'identification a donc permis une analyse méthodologique des différentes façons par lesquelles la constitution de l'être sexué est marquée et oscille entre ces deux pivots.

Le texte est structuré en quatre discussions. Chacune est articulée autour d'une conception différente de la notion d'identification chez Lacan, laquelle renvoie à l'une des quatre instances du nœud borroméen, présentées dans le séminaire « R.S.I. ».

Le premier temps explore les points centraux des dites formules de la sexuation à partir de Lacan et de commentateurs, en démontrant leur limite par rapport aux questions de genre. Ensuite on propose une nouvelle lecture du séminaire "Les non-dupes errent», qui permet d'ériger la proposition axiale de notre thèse selon l'axiome "l'être sexué ne s'autorise que de lui-même et de quelques autres ", dans la mesure où il présente et condense une théorie de l'identification sexuée qui non seulement suit des positions présentées auparavant (comme la critique de l'aristotélisme et la proposition du pas-tout) mais encore apporte de nouveaux éléments (l'acte, l'importance des autres, l'émancipation du dualisme des jouissances à partir d'une lecture borroméenne) aux coordonnés par lesquelles Lacan pensait classiquement la différence sexuelle.

Le contexte d'émergence de cette nouvelle lecture de la sexuation fournit des bases pour penser un processus d'identification se concrétisant dans la tension entre un choix du sujet et son insertion dans une groupe. De plus, le développement de cette autre perspective permet de déceler, dans les derniers séminaires, une théorie de l'identification jusqu'alors ignorée, celle de l'identification au "groupe », distingué de la «foule» freudienne, dans la mesure où Lacan assure qu'« un groupe, c'est réel ».

AMBRA, P. « Des formules au nom : bases pour une théorie de la sexuation chez Lacan ». In Analysis, v. 2, p. 69-70, 2018. Doi: 10.1016/j.inan.2018.02.006 
Ainsi avons-nous rassemblé plusieurs définitions du réel, se situant au-delà de la différence sexuelle binaire pour, à l'aide de la notion de lettre, analyser ladite prolifération identitaire supposée dans les mouvements LGBTTQQIAAP $+{ }^{1}$. Force est de constater que loin de constituer des défenses phalliques contre le réel de la différence sexuelle - cette inscription vise justement l'impossible du sexuel en instituant un horizon de contingence, étant donné que, à la limite, de nouvelles lettres sont toujours prêtes à surgir et à se réarranger, dans un mouvement échappant tant aux lois symboliques qu'aux fixations imaginaires. Cette idée se soutient, d'un côté, de la proposition selon laquelle le réel émerge historiquement et, de l'autre, de l'exposition du caractère d'indétermination dans ce quelques, qui trouble la consistance de l'ensemble «autres ». Ainsi, en concevant l'identification sexuée par cette visée du réel, propose-t-on que le sujet s'identifie aussi au dit trou du groupe et à l'horizon de son incomplétude.

Le deuxième chapitre s'axe ensuite autour de la notion d'identification symbolique. Lacan a proposé, au cours de son virage structuraliste, une association forte entre la différence sexuelle et la notion de signifiant. De ce fait, " homme » et " femme » ne seraient que des signifiants, ayant des effets de signification par leur opposition réciproque et complémentaire. Toutefois, en reprenant l'idée de système symbolique saussurien et les définitions ultérieures de signifiant chez Lacan, il nous est apparu important de tisser un autre réseau conceptuel pour les identifications sexuées. Nous avons essayé de démontrer que la naissance de la notion de " grand Autre » a effectivement eu lieu à partir de l'idée de " grands Autres », soit d'une altérité symbolique certes, mais plurielle.

$\mathrm{Au}$ vu tant des spécificités des constitutions de nouvelles identités de genre que de la notion lévi-straussienne de "norme » au cœur de la pensée structuraliste de Lacan, nous avons repris la discussion à propos des « normes » selon une autre perspective. Considérant la distinction entre "normativité » et "normalisation » chez Canguilhem, on a défini la normalisation comme le caractère social de l'imposition des limites de variabilité et la normativité comme une activité vitale d'instauration de nouvelles conditions de vie. La construction de cette rationalité, plaçant la possibilité des variations au cœur de la structure, converge avec notre lecture du rapport entre loi et norme chez Lévi-Strauss (pour qui la seule loi universelle de la parenté serait celle qui reconnaît que toute société dispose de normes sociales distinctes pour la formation d'alliances), avec l'idée de constellation symbolique (qu'on peut résumer par l'idée que le contenu de chaque élément du système est fixé par la position qu'il occupe relativement aux autres) et avec celle, enfin, du complexe comme structure aux contenus variables.

Les commentaires de Judith Butler et Michel Foucault sur Herculine Barbin ont été relus pour exemplifier comment - en brisant la normativité vitale qui localisait Herculine dans la constellation de ses autres - une normalisation médico-juridique instaure une souffrance d'indétermination causée par le bouleversement de l'identification qui garantissait les conditions d'intelligibilité de ses liens sociaux.

La section finale de ce chapitre propose une discussion sur les notions d'altérité chez Butler et Laplanche et leurs dialogues possibles avec Lacan. Laplanche nous invite à concevoir l'altérité à laquelle l'enfant répond comme formée par les socii, soit le noyau de

\footnotetext{
${ }^{1}$ Lesbiennes, gays, bisexuels, transgenres, transsexuels, queer, en questionnement, intersexes, asexués, alliés, pansexuelles. Le «+ » est rajouté par les mouvements pour souligner d'autres possibles identités, encore méconnues.
}

AMBRA, P.. « Des formules au nom : bases pour une théorie de la sexuation chez Lacan ». In Analysis, v. 2, p. 69-70, 2018. Doi: 10.1016/j.inan.2018.02.006 
base de la sociabilité, pour Freud, dans Psychologie des Masses constitué de petits autres. Cette altérité radicale, autant que plurielle, est aussi discutée par Butler et celle-ci rejoint l'hypothèse de ce que l'être sexué ne s'autorise ni de la présence/absence du phallus ni de l'identification cisgenre à un des parents, mais plutôt d'un complexe de genre où le caractère symbolique, décentré et constellé de l'altérité, est fondamental.

Dans un troisième temps de la thèse, la construction de la centralité du semblable dans le processus de sexuation nous conduit au complexe d'intrusion, localisé dans le passage du complexe de sevrage au complexe d'Edipe. Ce complexe intermédiaire est intimement lié au stade du miroir, où le semblable (le frère, l'ami et même sa propre image au miroir) joue un rôle essentiel dans l'avènement du moi. Dès lors, nous avons formalisé une définition de l'assomption comme acte de prendre comme «soi » ce modèle venant de l'autre, dans le contexte de la sexuation d'un corps entier. Autrement dit, à l'opposé d'une théorie de la sexuation supposant que l'identité serait donnée par une construction basée sur l'organe génital, nous avons proposé de concevoir la sexuation comme un processus où l'identification prend place dans une dialectique allant du zéro au deux, pour pouvoir compter le moi comme un. Dit autrement, comme dans le stade du miroir, l'expérience du morcellement trouve sa fin et le moi de l'enfant n'advient qu'au moment où les semblables sont aussi reconnus en tant que tels.

L'incidence de ces quelques autres de la sexuation serait donc double : il s'agit autant de ces autres qui désignent le sujet, que des semblables auxquels le sujet va s'identifier horizontalement. La centralité du caractère collectif du processus d'identification peut être renforcée, selon nous, par le texte «Le temps logique et l'assertion de certitude anticipée », où nous trouvons la logique de l'assomption d'une identification du moi régie par un acte qui est singulier, mais, paradoxalement, ne peut se réaliser que collectivement. Étant donné, dans ce texte, l'échec d'une telle identification associée par Lacan à la barbarie, il nous a été possible de faire une liaison avec le type de souffrance en cause pour ceux qui vivent leurs sexuations comme échappant à la normalisation sociale.

Nous avons aussi exploré le caractère central de l'expérience de jubilation dans l'assomption, par la discussion de son rapport avec la «lalangue » et la notion de « jouissance de l'Autre », considérant que cette dernière se localise entre l'imaginaire et le réel. C'est-àdire que, plus précisément qu'une jouissance dite féminine, la spécificité de la jouissance de l'Autre dans ce processus, pourrait être vu justement comme la promotion de l'unification du corps et du moi par la présence de cet Autre (ou autres) qui nomme(nt) et joui(ssent) avec l'infans de sa reconnaissance comme identité sexuée.

La quatrième section du texte est organisée autour de la notion de nomination. Nous rapportons la thèse lacanienne décrivant l'acte lié à la nomination comme quatrième nœud qui unifierait les trois registres. Nous analysons un cas qui condenserait les points capitaux de notre théorie de la sexuation, un entretien mené par Lacan avec un sujet trans enfermé. Son récit semble clarifier la manière dont la nomination fournit un modèle de la théorie du processus d'identification sexuée, grâce à l'autorisation de soi-même et quelques autres. Notre analyse a présenté aussi des éléments pour une réflexion sur l'importance du nom propre pour les minorités sexuelles, aussi bien que du concept de signature chez Derrida.

Pour conclure, grâce à une révision critique des commentateurs tenant la notion de sinthome comme paradigme de la transsexualité psychopathologiquement conçue et par notre constatation de l'importance du nom dans le nouage des trois registres, la différence entre les

AMBRA, P.. « Des formules au nom : bases pour une théorie de la sexuation chez Lacan ». In Analysis, v. 2, p. 69-70, 2018. Doi: 10.1016/j.inan.2018.02.006 
sexuations cis et trans est repensée, car dans les deux cas il s'agit d'une prise en charge du sujet de la nomination qui vient d'ailleurs et le situe face à quelques autres. Tous les êtres qu'ils soient minorisés ou non - pourraient être pensés ainsi comme sexués par l'autorisation « de soi-même et de quelques autres ». Les différences de souffrance psychique pourraient à ce compte être repensées non plus en termes de «psychose » ou " perversion », ou par rapport à l'anatomie, mais en fonction de l'extension de la capacité du sujet à se nommer et à être nommé à l'intérieur d'une société aux paramètres de normalisation hétérocentrés. Bref, par notre travail, nous soumettons à débat une cartographie des bases épistémologiques par lesquelles on pourrait concevoir une nouvelle notion de sexuation chez Lacan.

\section{Remerciements}

Je tiens à remercier la CAPES (Coordenação de Aperfeiçoamento de Pessoal de Nível Superior) et le CNPq (Conselho Nacional de Desenvolvimento Científico e Tecnológico) pour le financement de cette recherche.

AMBRA, P.. « Des formules au nom : bases pour une théorie de la sexuation chez Lacan ». In Analysis, v. 2, p. 69-70, 2018. Doi: 10.1016/j.inan.2018.02.006 